\title{
Quattro forme di enciclopedia ovvero le metamorfosi dell'albero
}

Aprendo un dizionario della lingua italiana (per esempio il DevotoOli), si trova questa definizione di "enciclopedia":

Ampia pubblicazione che si prefigge di raccogliere e trattare ordinatamente e per quanto possibile esaurientemente le cognizioni relative alle scienze e alle arti nel loro complesso, o limitatamente a uno o più determinati campi dello scibile. . . .

La definizione sembra corrispondere abbastanza bene al concetto editoriale che oggi abbiamo di enciclopedia, sia essa l'Enciclopedia Treccani o una enciclopedia "professionale," di agraria o di scienze giuridiche. Ma lo stesso dizionario aggiunge subito dopo una definizione che si riferisce alla accezione "arcaica" del termine:

Sistema completo di cognizioni che tenda ad abbracciare l'intero scibile o si limiti a campi e argomenti specifici ...

aggiungendo una definizione del termine medievale encyclaopedia, come erratamente tradotto dal greco enkyklios paideia, e cioè educazione circolare, e quindi completa (che è poi una definizione derivata da Plinio il Vecchio).

Diciamo subito che è l'accezione arcaica che qui ci interessa, perché è la piú moderna. Essa ripropone ancora oggi la domanda sulle possibilità d'unificazione del sapere. L'enciclopedia, in questo senso, è dunque l'idea regolativa (che può dar luogo sia a una ipotesi di ricerca, a un tentativo di costruzione, o alla dimostrazione della sua impossibilità teorica o pratica) di un sistema e di un quadro che unifichi tutte le conoscenze di una determinata epoca. Di questa utopia le enciclopedie editoriali sono naturalmente delle pallide approssimazioni, talora delle parodie. Piú spesso sono dei semplici vocabolari che chiariscono il significato immediato e ristretto di un dato termine senza preoccuparsi di stabilire connessioni o di individuare contraddizioni. 
Quella che qui ci interessa è l'idea detta "araldica" di enciclopedia perché essa è nel contempo la sua idea forte. La linea forte-debole stabilisce ovviamente un continuum di possibilità di cui l'estremo piú debole è il dizionarietto a uso del turista, mentre il massimo della forza si identifica con una idea della mente divina simile a quella descritta da Dante nel Paradiso, XXXIII, 85-87:

Nel suo profondo vidi che s'interna

legato con amore in un volume

ciò che per l'universo si squaderna

Il che equivale a dire che l'idea fortissima di enciclopedia presuppone l'esistenza di un dio onnisciente, ed onnisciente circa un universo che egli stesso ha ordinato secondo ragione. Per cui, come ci si appressa ai limiti estremi del continuum forte-debole, da un lato abbiamo una metafisica della Rapprensentazione Adeguata (il mondo è complesso ma ordinato e noi abbiamo la possibilità di conoscerlo e rappresentarlo nella sua complessità e nel suo ordine) e dall'altro una sorta di empirismo radicale, di relativismo assoluto, per cui il mondo altro non è che un pulviscolo di entità non correlate e in ogni caso non correlabili, cosí che dell'una non si può dare definizione in termini dell'altra.

L'umanità ha giocato, in epoche diverse, su diversi concetti di enciclopedia. Volendo però elaborare alcune schematizzazioni che ci permettano di stabilire il senso che possa avere oggi per noi la nozione di enciclopedia, ci si potrà riferire a una rosa di quattro possibilità, ciascuna delle quali non corrisponde a una impresa editoriale precisa, né a una precisa posizione filosofica, ma piuttosto circoscrive, molto in generale, un atteggiamento verso l'impresa enciclopedica, un modo di avvicinarsi all'enciclopedia, o meglio ancora, un'ampia struttura soggiacente che distingue, attraverso la varietà degli esiti specifici, tre ideologie dell'enciclopedia.

a) L'enciclopedia a cumulo. Non si hanno idee chiare su quale sia la forma del mondo. Si accumulano tutte le informazioni a disposizione sul mondo. Siccome non si posseggono ipotesi sulla forma del mondo, non si hanno criteri per distinguere le informazioni vere da quelle false. Ma una sorta di fiducia nella verità del mondo e nella veridicità di tutte le autorità che han parlato sul mondo, incita a ritenere che ogni informazione sia vera. Questo tipo di enciclopedia, dal punto di vista dell'uomo moderno, appare come una raccolta acritica di assurdità mescolate a qualche osservazione attendibile. 
b) L'enciclopedia ad albero. Si ha una idea precisa circa la forma del mondo. Si accumulano tutte le informazioni a disposizione e le si fanno rientrare nel quadro di questa forma del mondo. Non ci si chiede ancora se siano vere o false (secondo $i$ criteri odierni di verificabilità scientifica) ma semplicemente se si inseriscano in qualche modo nel quadro, e vi stiano a proprio agio. Mentre la possibilità numero 1 non si pone neppure il problema della contraddittorietà delle informazioni, questa possibilità numero 2 cerca di individuare le contraddizioni e di comporle: non di eliminarle, bensí di spiegare, nei termini del quadro di riferimento, perché esse apparentemente sussistano.

c) L'enciclopedia illuminista (albero/mappa). Si pronuncia una ipotesi sulla forma del mondo e ci si propone un quadro di riferimento, non necessariamente esplicito. Alla luce di questo quadro si decide quali siano le informazioni vere e quelle false, e quali le informazioni rilevanti e quali le impertinenti. Questa enciclopedia numero 3 non è meno "ideologica" delle altre due, ma presenta un tratto che la differenzia dalle altre: essa è conscia del proprio formato ideologico, ne confessa i presupposti. Di solito, proprio in virtù del progetto che la muove, essa non pretende di registrare soltanto il mondo cosí come è, ma si presenta come una proposta di riorganizzazione di quel mondo, già rappresentato dalla enciclopedia ad albero.

d) L'enciclopedia semiotica (mappa/rizoma). Si possiede una quantità enorme di informazioni. Tutte sono verificabili non nel senso che si sappia se siano vere o false, ma nel senso che si sa come sono state prodotte: di alcune si sa che provengono da operazioni dette "scientifiche" e che quindi, nei termini della concezione odierna della scientificità, son ritenute "vere"; di altre si sa di preciso che sono immaginarie, prodotti deliberati di finzione; di altre ancora non si sa con esatezza come etichettarle dal punto di vista della verità, ma se ne riconosce l'esistenza sociale sotto forma di "opinioni" circolanti. Ma proprio in virtù dell'abbondanza e della sconnessione di queste informazioni, non si può piú pretendere di organizzarle in un quadro o secondo una forma. Si postula pertanto un sistema di interrelazioni tra dati che non abbia l'aspetto di un quadro e non sia formato, ovvero non assuma l'aspetto delle forme tradizionalmente riconosciute come tali. Dato che il progetto enciclopedico prevede che in qualche modo i dati siano correlati, una organizzazione ci dovrà pur essere. Ma questa enciclopedia elabora una sua idea di organizzazione disorganizzata, o di forma informe, o di plurità e coesistenza delle forme. Natural- 
mente, l'abbondanza stessa dei dati, impedisce di costruire questa enciclopedia come un oggetto finito. Essa appare piuttosto come un progetto aperto: non una utopia come terminus ad quem, e cioè uno stato di perfezione da raggiungere, ma una utopia come idea regolativa, come progetto ante quem, la cui forza è data proprio dal fat to che esso non puo e non deve essere realizzato in modo definitivo.

Ci pare che il fare e pensare enciclopedia, nel corso dei secoli, si sia mosso tra queste quattro possibilità.

\section{La prima forma, il cumulo.}

In periodo ellenistico, tra la crisi del paganesimo, l'apparizione di nuovi culti, i primi tentativi di organizzazione teologica del cristianesimo, appaiono le enciclopedie a cumulo, il cui modello sopravviverà per tut to il medio evo e anche dopo. Esempio tipico di questa enciclopedia è il Physiologus, composto in greco in ambiente siriano o egiziano tra il II e il IV secolo d.C., e poi tradotto e parafrasato in latino (oltre che in etiopico, in armeno, in siriaco). Dal Fisiologo derivano tutti i bestiari medievali e per tutta l'età media le enciclopedie si ispirarono a questa fonte.

Il Fisiologo non ha logica: ha, si direbbe, una retorica. Nel senso in cui, per Aristotele, l'argomentazione retorica doveva partire da premesse probabili, e una premessa era probabile in quanto rappresentava una opinione corrente, condivisa dai più. Il Fisiologo raccoglie tutto quello che è stato detto intorno agli animali veri o presunti. Si potrebbe pensare che parla con proprietà di quelli noti al suo autore, e con incontrollata fantasia di quelli che egli conobbe per sentito dire, in una parola che sia preciso circa la cornacchia e impreciso circa l'unicorno. Invece è preciso, quando ad analisi delle proprietà, rispetto a entrambi, e inattendibile in entrambi i casi. Il Fisologo non stabilisce differenze tra il noto e l'ignoto. Tutto è noto in quanto alcune lontane autorità ne hanno parlato, e tutto è ignoto perché fonte di meravigliose scoperte, e chiave di volta di recondite armonie.

La verità è che il Fisiologo ha una sua idea della forma del mondo, per quanto vaga: tutti gli esseri del creato parlano di Dio. Pertanto ogni animale deve essere visto, nella sua forma e nei suoi comportamenti, come simbolo di una realtà superiore:

I ricci hanno la forma di una palla e sono tutti ricoperti di aculei. Il Fisiologo ha detto del riccio che si arrampica sulla vite e va dove c'è l'uva, e getta per terra i chicchi e vi si rotola sopra, e i chicchi si conficcano nei suoi acueli, ed esso li porta ai figli lasciando il tralcio spoglio. 
Perché al riccio viene attribuita questa bizzarra abitudine? Per trarne una acconcia spiegazione morale: il fedele deve rimanere aggrappato alla Vite spirituale senza permettere che lo spirito del male vi si arrampichi e lo renda spoglio di ogni grappolo. È ovvio che un altro animale, in luogo del riccio, potrebbe assolvere allo stesso compito simbolico. Le altre enciclopedie posteriori che, sul modello del Fisiologo, descrivono animali reali e fantastici, complicano questo gioco di riferimenti simbolici, sino ad entrare in mutua contraddizione; ma sopravvengono altre enciclopedie che non esitano a registrare sensi contraddittori. Il leone può essere sia simbolo di Gesù che simbolo del diavolo. In quanto nasconde con la coda le tracce che lascia sulla polvere per ingannare i cacciatori, è simbolo di redenzione dai peccati; in quanto risuscita col suo fiato il leoncino nato morto, entro il terzo giorno, è simbolo della risurrezione; ma in quanto Sansone e Davide lottano contro un leone di cui aprono le mascelle, è simbolo della gola dell'Inferno, e il Salmo 21 canta appunto "salvia me de ore leonis." Chi deciderà quale sia la retta interpretazione dell'animale-segno in un determinato contesto? "L'auctoritas," rispondono gli enciclopedisti medievali, la tradizione, le analisi già condotte dai padri e dai dottori.

Cosí l'enciclopedia a cumulo, appunto, accumula, e lo fa anche quando pare organizzare la sua selva di informazioni secondo un disegno. Nel VII secolo le Etimologie di Isidoro di Siviglia appaiono suddivise in capitoli, ma il criterio che regge la suddivisione è del tutto occasionale. L'inizio pare ispirato alla divisione delle arti (grammatica, dialettica, retorica, matematica, musica, astronomia) ma poi segue, al di fuori del Trivio e del Quadrivio, la medicina, quindi si passa a considerare la legge e i tempi, i libri e gli uffici ecclesiastici, Dio e gli angeli, la chiesa, le lingue, i rapporti di parentela, i vocaboli strani, l'uomo e i mostri, gli animali, le parti del mondo, gli edifici, i campi, le pietre e i metalli, l'agricoltura, la guerra e i giochi, le navi, i vestiti, gli strumenti domestici e rustici. La divisione è chiaramente disorganica e fa venire in mente la ormai classica tassonomia impropria di Borges:

gli animali si dividono in (a) appartenenti all'imperatore, (b) imbalsamati, (c) addomesticati, (d) maialini di latte, (e) sirene, (f) favolosi, (g) cani in libertà, (h) inclusi nella presente classificazione, (i) che si agitano follemente, (j) innumerevoli, (k) disegnati con un pennello finissimo di pelo di cammello, ( $\mathrm{l}$ et coetera, $(\mathrm{m})$ che fanno l'amore, ( $\mathrm{n}$ ) che da lontano sembrano mosche ...

e se le suddivisioni di Isidoro sembrano piú ragionevoli di quelle di Borges, si veda poi come a loro volta si suddividono: perché mai nel capitolo su navi, edifici e vestiti devono apparire i paragrafi sui 
mosaici, e sulla pittura; e perché la parte sugli animali deve dividersi in Bestie, Animali piccoli, Serpenti, Vermi, Pesci, Uccelli e Piccoli animali alati?

L'enciclopedia a cumulo appartiene a un'epoca che non ha ancora trovato una immagine definitiva del mondo; per questo l'enciclopedista raccoglie, enumera, addiziona, spinto soltanto dalla curiosità e da una sorta di umiltà antiquaria. L'enciclopedia a cumulo ha questo di comune con l'enciclopedia semiotica: è fedele a quanto è stato già detto.

\section{La seconda forma, l'enciclopedia ad albero.}

La seconda forma, ancora medievale, almeno alle origini, nasce da una ipotesi piú precisa, seppure del tutto astratta e teorica, sul sistema del sapere. Un modello del genere potrebbe essere, nel XIII secolo, il triplice Speculum Mundi di Vincenzo di Beauvais (Speculum Doctrinale, Historiale, Naturale), che ha già l'organizzazione di una Summa scolastica. La Summa theologiae di Tommaso d'Aquino ha una struttura ferrea, è un albero (gerarchizzato nei suoi rami principali e nelle loro derivazioni), con la radice all'in su, che parte da Dio come causa esemplare, e in se stesso e nelle sue creature, rispetto a cui appare come causa efficiente, finale e riparatrice, poi si dirama nella considerazione delle nature angeliche, del mondo e dell'uomo, e rispetto all'uomo esamina gli atti, le passioni, gli abiti e le virtù, passando poi ai misteri dell'incarnazione e ai sacramenti come strumento continuo di rendenzione, per terminare ai novissimi come vestibolo della vita eterna. Dove le fronde estreme riconducono per cosí dire alla radice, l'intero universo è suddiviso dall'essere perfettissimo sino ai meno perfetti, ma in modo che la suddivisione anziché una gerarchia statica rapprensenti anche un itinerario, a Dio come origine a Dio come fine. In questo albero tutti i problemi minori trovano il loro posto, e nulla puo apparire in mutua contraddizione, tutto deve comporsi in un disegno omogeneo. Anche se Vincenzo di Beauvais era dotato di minor originalità speculativa di quanto non fosse il dottore d'Aquino, il suo Speculum rivela una pari energia classificatrice.

Si veda l'indice dello Speculum naturale: qui la suddivisione non è ispirata a un criterio filosofico o a una tassonomia statica, ma a una scansione storica, che segue i giorni della creazione: primo giorno il Creatore, il mondo sensibile, la luce; secondo giorno il firmamento e i cieli; e cosí via, per arrivare agli animali, alla formazione del corpo umano e alla storia dell'uomo.

In queste enciclopedie lo schematismo agisce da riduttore sulla ricchezza delle informazioni (e molti dati leggendari vengono 
lasciati cadere), ma garantisce che le informazioni non entrino in contraddizione reciproca. Molte enciclopedie rinascimentali e barocche sono della stessa natura. Stuttura portante di queste enciclopedie è l'indice, e l'indice, come si è detto, è ad albero. Al massimo si tratterà di trovare una logica sempre piú sottile dell'albero, come farà Raimondo Lullo (e come tenterà in misura piú astratta di fare Leibniz), attraverso l'elaborazione di un'arte combinatoria che, prevedendo e generando tutte le combinazioni possibili, permetta una rapprensentazione sempre piú pefettamente gerarchizzata e sempre piú esaustiva. Queste enciclopedie sono a modo proprio dei cervelli elet tronici ante litteram, e procedono come i cervelli elettronici detti digitali, per disgiunzioni binarie. Il modello, semplificando all'estremo, è il seguente:

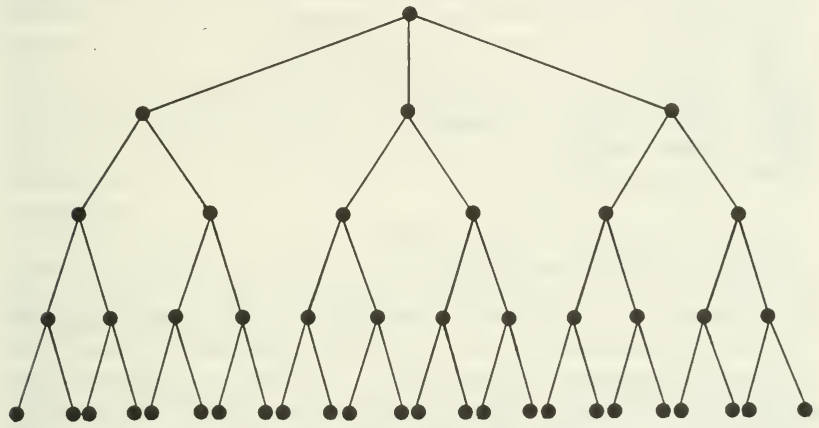

3. La terza forma, l'enciclopedia illuminista. Dall'albero alla mappa.

L'enciclopedia di terza forma non è necessariamente diversa, quanto a struttura gerarchica, da quella ad albero di seconda forma. Ciò che la differenzia è in primo luogo la confessa natura ipotetica dell'albero: esso non riproduce una presunta struttura del mondo, ma si presenta come la soluzione piú economica per affrontare e risolvere un certo problema di riunificazione del sapere; in secondo luogo l'enciclopedia sa che l'albero organizza ma impoverisce, e si ripromette di individuare quanto piú può i percorsi intermedi tra $\mathrm{i}$ vari nodi dell'albero, cosí che a poco a poco esso si trasformi in una carta geografica ovvero in una mappa. Ora una mappa non è un albero: la carta d'Italia non stabilisce nessuna priorità tra Torino e Milano, traccia solo un reticolo di connessioni (strade, fiumi, ferrovie) tra i nodi principali, ma lascia libero l'occhio o il viaggiatore, di partire da Torino per andare a Milano o viceversa. 
Non solo, ma anche se rappresenta Bologna piú importante (come nodo di strade o come grandezza) di Rimini o di Arezzo, non impedisce a chi sia interessato al tragitto Rimini-Arezzo di percorrerlo senza triangolare su Bologna (perché Bologna non è essenziale alla definizione delle altre due città, non si pone come principio generativo del tragitto Rimini-Arezzo).

Il modello dell'enciclopedia illuministica si regge, è vero, su una sorta di falsariga ad albero, piú o meno di questo tipo:

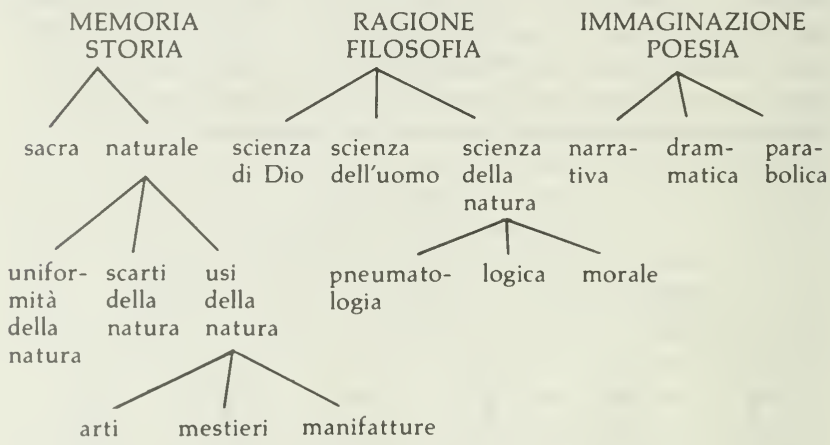

Ma d'Alembert, nel Discorso preliminare all'Enciclopedia, mentre forniva informazioni sui criteri di organizzazione dell'opera, che peraltro non potevano essere visibili, a causa della riorganizzazione alfabetica ( $\mathrm{e}$ in questo l'enciclopedia di terzo tipo si distingue da quello di secondo tipo, ed è da un lato meno immediatamente repressiva, dall'altro piú sottilmente ideologica) sviluppa da un lato la metafora dell'albero e dall'altra la mette in questione, parlando invece di "mappamondo":

non ci resta che formare un albero genealogico e enciclopedico. ... Spiegheremo in un momento l'uso che intendiamo fare di quest'albero: il farlo, invece, non è senza difficoltà. . . . Il sistema generale delle scienze e delle arti è una specie di labirinto, di cammino tortuoso che lo spirito affronta senza troppo conoscere la strada da seguire... Ma questo disordine, per quanto filosofico sia per la mente, sfigurerebbe, o almeno annienterebbe del tutto un albero enciclopedico nel quale lo si volesse rapprensentare. D'altra parte, come già abbiamo rilevato a proposito della logica, la maggior parte delle scienze che consideriamo come racchiudenti in sè i principi di tutte le altre, e che per questa ragione devono occupare il primo posto nell'ordine enciclopedico, non tengono il primo posto nell'ordine genealogico delle idee, perché non sono state inventate per prime. ... Infine il sistema delle nostre conoscenze è composto di diverse branche, di cui molte hanno uno stesso punto di riunione; e poiché 
partendo da questo punto non è possibile imboccare contemporaneamente tutte la vie, la determinazione della scelta risale alla natura dei diversi spiriti. .. La stessa cosa non avviene invece per l'ordine enciclopedico delle nostre conoscenze. Quest'ultimo consiste nel riunirle nel piú breve spazio possibile, e nel porre, per cosí dire, il filosofo al di sopra di questo vasto labirinto, in un punto di vista molto elevato da dove gli sia possibile scorgere contemporaneamente la scienza e le arti principali; vedere con un sol colpo d'occhio gli oggetti delle sue speculazioni e le operazioni che può fare su questi oggetti; distinguere le branche generali delle conoscenze umane, i punti che le separano o che le accomunano, e intravvede persino, a volte, le vie segrete che le riuniscono. È una specie di mappamondo che deve mostrare i principali paesi, la loro posizione e le loro vicendevoli dipendenze, il cammino in linea retta che v'è dall'uno all'altro; cammino spesso interrot to da mille ostacoli, che non possono essere noti in ciascun paese che agli abitanti e ai viaggiatori, e che non potrebbero essere mostrati che in carte particolari molto minute.

Queste carte particolari saranno i diversi articoli della Enciclopedia, e l'albero o sistema figurato ne sarà il mappamondo. Ma, come nelle carte generali del globo da noi abitato, gli oggetti sono piú o meno accostati tra loro, e presentano un colpo d'occhio diverso a seconda del punto di vista da cui si è posto il geografo che costruisce la carta, cosí pure la forma dell'albero enciclopedico dipenderà dal punto di vista da cui ci porremo per guardare l'universo della cultura. Si possono dunque immaginare tanti diversi sistemi della conoscenza umana quanto mappamondi di diverse propiazioni. ...

Spesso un oggetto che, per una delle sue proprietà, è stato messo in una classe, appartiene a un'altra classe per altre sue proprietà ed avrebbe potuto esservi posto altrettanto bene. Necessariamente, dunque, la divisione generale mantiene dell'arbitrario. ...

Segue poi l'esposizione del criterio seguito e dell'albero adottato: denunciato chiaramente come arbitrario. Come se, si suggerisse, dato che il mappamondo (o il globo) andava percorso, gli autori si fossero fatti un itinerario, consci di non esaurire con questo le possibilità di esplorazione e i tragitti che la natura del globo consente.

Queste pagine di D'Alembert fanno qualcosa di piú che introdurre il lettore allo spirito dell'enciclopedia illuminista (di terzo tipo): di fatto preludono alla discussione dei problemi che debbono necessariamente portare alla enciclopedia di quarto tipo. Infatti in esse si disegna con estrema chiarezza l'idea che il sistema enciclopedico non abbia centro.

\section{La quarta forma, l'enciclopedia semiotica. Dalla mappa al rizoma.}

La quarta forma di enciclopedia non costituisce né un oggetto né un progetto editoriale. Ovvero, costituisce la totalità dei progetti editoriali. Ciò significa che la quarta forma di enciclopedia è già 
scritta, è in corso di scrittura, sarà scritta, ma nessuno può proporsi di scriverla. Essa si identifica con la biblioteca delle biblioteche. Se ce ne occupiamo in questa sede è perché il termine "enciclopedia" è oggi usato come preciso concetto della semantica (e della semiotica in generale) ed è usato nel modo in cui diremo proprio perché è entrata in crisi una nozione di enciclopedia a albero.

Il problema nasce nelle discussioni contemporanee di semantica quando si tratta di costruire un codice, ovvero un sistema di equivalenze che facciano corrispondere a delle espressioni fisiche (parole, od altri segni in sistemi semiotici non verbali) una data porzione di contenuto (o significato).

Un dizionario bilingue associa a termini di una lingua $\mathrm{A}$, termini di una lingua $B$. Cosí facendo non spiega il significato dei termini dell'una o dell'altra lingua, se non ricorrendo al piú elementare e discutibile dei criteri semantici, la sinonimia: / cane / in italiano è sinonimo di "chien" in francese.

Un vocabolario di una lingua invece costituisce un dato termine non solo con sinonimi, ma anche con definizioni: / cane / significa "mammifero domestico dei carnivori, onnivoro, con odorato eccellente, pelame folto di vario colore, pupilla rotonda, dimensioni, forma del muso e attitudini variabili secondo la razza." Cosí per esempio lo Zingarelli. E se si limitasse a questo, il vocabolario sarebbe abbastanza insufficiente. Per esempio mi direbbe cos'è un cane secondo la zoologia (mammifero carnivoro) ma non $\mathrm{mi}$ insegnerebbe a riconoscere un cane. In effeti, il vocabolario citato aggiunge delle informazioni visive e cioè una serie di illustrazioni che mostrano trenta tipi di cani diversi. Cosí facendo evita di specificare verbalmente alcune proprietà di questi animali, per esempio che hanno quattro gambe, una coda, eccetera. In piú il vocabolario aggiunge alcune espressioni fatte, brevi frasi, nomi composti (come / cane da pagliaio /, / mangiare come un canel) che costituiscono esempi di uso linguistico e aiutano in qualche modo a circoscrivere meglio il significato del termine in questione.

La semantica strutturale contemporanea ha stabilito che il significato di una espressione debba essere analizzato in unità semantiche elementari, dette anche "semi," ovvero proprietà, e le varie teorie auspicano o propongono (sempre in modo ancora insoddisfacente) che questi semi siano organizzati in qualche modo, dai piú generali ai piú specifici, e possano dar ragione di tutti i possibili usi linguistici del termine. In altre parole, si auspica una semiotica componenziale (che analizza cioè le componenti semantiche, ovvero i semi, del semema corrispondente a un dato termine - il semema altro non essendo che lo spettro organizzato di tutte le 
proprietà o semi) che serva come sistema o serie di istruzioni per inserire correttamente il termine nei contesti appropriati.

Infatti l'analisi in proprietà semantiche dovrebbe permetterci di riconoscere le espressioni semanticamente ben formate da quelle semanticamente devianti. Supponiamo che un termine, per esempio / scapolo / (per prendere uno degli esempi piú trattati dalla logica, dalla filosofia del linguaggio e della semantica dell'ultimo secolo) possa essere analizzato in queste proprietà semantiche elementari: uomo, maschio, adulto, non sposato.

Ecco che questo sistema di proprietà permetterebbe di riconoscere come semanticamente deviante la frase "la madre di Luigi è scapolo," che violerebbe regole di amalgama tra/madre/e / scapolo/ dato che / madre/ dovrebbe essere analizzato come "uomo, femmina, adulta" (non è necessario che una madre sia sposata. ..." Due proprietà di / madre/ non si amalgamerebbero con due proprietà di / scapolo/.

Se fosse possibile analizzare tutti i termini in un numero cosi ridotto di proprietà, non esisterebbero problemi per una semantica componenziale (cosí come non esisterebbero per un cervello elettronico capace di tradurre da una lingua a un'altra o di formulare parafrasi di espressioni linguistiche). Ma il problema sorge quando ci si trovi di fronte a una frase come: "quello scapolo ha tre gambe." Dovremmo dire che poiché uno scapolo è "uomo" (e postulando che anche le proprietà semantiche siano a loro volta analizzabili semanticamente) e gli uomini hanno la proprietà di avere due gambe, la frase in questione è semanticamente anomala.

Ma ci si avvede subito di un inconveniente. Che uno scapolo sia uomo adulto e non sposato non è tanto una "verità" naturale, quanto una convenzione semantica, o meglio un postulato di significato. Nell'accettare il codice di una data lingua si decide di usare il termine / scapolo/ solo per gli essere umani, adulti e non sposati. Una volta presa questa decisione sarà scorretto usare / scapolo/ per una donna (che sarà piuttosto / nubile/) o per un bambino (visto che / scapolo/ viene scelto per indicare la condizione di non sposato di un adulto, e nessuno chiamerebbe scapolo un bambino se non per scherzare o per indicare con una figura retorica il fatto che, ovviamente, i bambini non possono sposarsi salvo condizioni particolari in civiltà o periodi storici specifici). Che invece un uomo abbia due gambe sembra una verità di fatto. E comę tutte le verità di fatto può essere negata o sospesa: niente vieta che con la frase "quello scapolo ha tre gambe" io voglia indicare un essere umano abnorme, un fenomeno da baraccone, che peraltro rimane pur sempre un essere umano. Cosí, tornando all'esempio di / cane /, sembra difficile (dati i postulati di significato 
posti dalla moderna zoologia) dire "quel cane non è un mammifero" mentre sembra possibile dire "quel cane non ha la coda" oppure "quel cane ha solo tre gambe."

La differenza è di tipo logico. In una gerarchia di postulati di significato il cane è inserito necessariamente nella classe dei carnivori e questi nella classe dei mammiferi, cosí che è impossibile che un animale sia cane senza essere carnivoro e mammifero. Questa relazione, per cui "se cane allora necessariamente mammifero" e "se questo animale non è un mammifero allora non può essere un cane" viene chiamata relazione di entailment, o come si usa ora tradurre in italiano, di implicitazione. L'implicitazione riguarda un insieme gerarchizzato di proprietà. La forma di questa gerarchia è ad albero, tanto è vero che trae la sua origine dal cosiddetto "albero di Porfirio," dovuto al filosofo neoplatonico del III secolo d.C. e commentatore di Aristotele. Porfirio organizza l'intero universo attraverso una scala o una struttura arborescente che procede per incassamento da genere a specie:

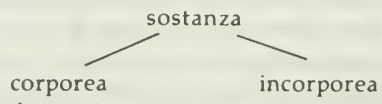

animata inanimata

sensibile insensibile

11

razionale irrazionale

/ \

Socrate, Platone. . :

$\mathrm{Si}$ vede facilmente che la relazione che lega i nodi inferiori dell'albero e quelli superiori è una relazione di entailment o implicitazione. Se Platone, allora sostanza razionale, se razionale allora sostanza animata, e cosí via. Non si puo dire di Platone (o di un uomo in genere) che sia sostanza razionale ma non sia animato.

Ora una semantica fondata solo su relazioni di entailment ha la possibilità di predire quali frasi siano ben formate e quali no, ma si trova a dover maneggiare solo poche proprietà, ciascuna delle quali rappresenta la classe generale entro cui rientra la proprietà sottostante. Cioè una semantica basata sull'entailment può dire che tutti gli scapoli sono non sposati e che non possono esserci degli sposati che sono scapoli, cosi come può dire che non possono esserci cani 
che non siano mammiferi, ma non può analizzare nei propri termini espressioni come "ho visto un cane che miagolava" o "non permetterò che mia figlia vada sola di sera a casa di uno scapolo." Infatti la prima frase presume, per essere giudicata in termini di correttezza semantica, che si sappia che i cani hanno la proprietà di abbaiare e che la proprietà di miagolare è tipica dei gatti; e la seconda prevede una serie di nozioni che chiameremo "sociali" sui rapporti di convenienza e sul fatto che di solito gli scapoli abitano soli, sono sospettati di condurre vita piú libera degli uomini maritati, eccetera eccetera - naturalmente nell'ambito di una capacità di comprendere le espressioni linguistiche (e il principio vale anche per i segni non verbali, sia pure in modi diversi) dipende proprio dalla nostra conoscenza di queste proprietà piú o meno "accidentali" e non "necessarie" e dall'intero sistema delle convenzioni dette extralinguistiche nell'ambito delle quali i termini linguistici vengono usati e si caricano di senso.

Ora vi è una corrente della semantica contemporanea che asserisce che l'analisi semica può essere fatta solo in termini di dizionario, e cioè ascrivendo a una data espressione le proprietà strettamente linguistiche; tutto il resto appartiene al sistema della conoscenza empirica e non fa parte di un dizionario, bensí di una enciclopedia. E siccome la rapprensentazione enciclopedica è potenzialmente infinita, non può costituire una base ragionevole per le rappresentazioni semantiche. Quale è la differenza tra dizionario ed enciclopedia? Su questo argomento sono stati spesi fiumi di inchiostro ma ci pare che la linea di discrimine tra i due concetti stia proprio nella relazione di entailment. Un dizionario in senso stretto deve solo tener conto dei termini impliciti. Nella definizione citata dallo Zingarelli, un cane è mammifero e carnivoro (e quindi per successive implicitazioni, animale ed essere organico) in virtù di postulati dizionariali di significato, mentre è solo in base a una conoscenza enciclopedica che ha odorato eccellente o pupilla rotonda (perché non si vede perché di fatto non possa esistere un cane con pupilla anormale e privo di odorato, mentre non ci può essere un cane che non sia mammifero).

Se è cosí, molte teorie che pretendono di fornire rappresentazioni semantiche in forma di dizionario, di fatto non rispettano i requisiti logici di un dizionario: e si veda proprio l'esempio, cosí abbondantemente fornito in molti testi, di / scapolo/. Infatti questo termine non può essere inserito in un solo albero di Porfirio che tenga conto delle quattro proprietà "uomo, maschio, adulto e non sposato." Se infatti la relazione discendente da generale a specifico è rappresentata come 


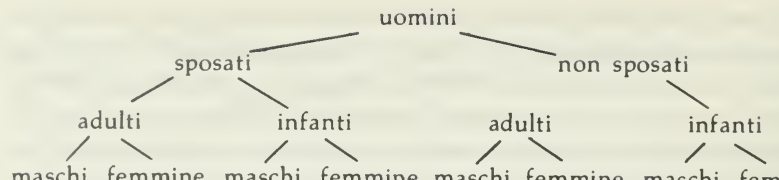

maschi femmine maschi femmine maschi femmine maschi femmine scapoli

uomo scapolo è necessariamente adulto maschio uomo e non sposato, ma non è vero che ogni maschio sia non sposato, come si vede dal diagramma. Né le cose cambierebbero se il diagramma fosse organizzato come segue:

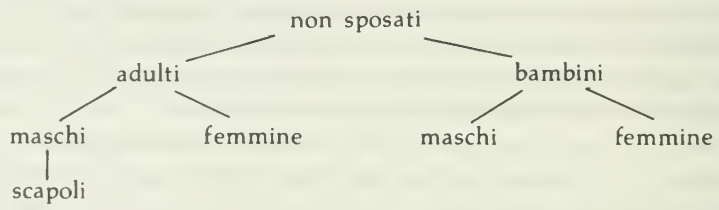

perché in tal caso tutti gli scapoli sarebbero necessariamente maschi adulti non sposati, ma non sarebbero necessariamente adulti tutti i maschi. Il che ci fa ricordare le parole di D'Alembert: che cioè gli alberi e le rappresentazioni gerarchizzate intervengono par dare un certo ordine alla varietà di una mappa, la quale però potrebbe supportare, da altri punti di vista, altri ordini e altri alberi. Infatti uno dei problemi discussi dai teorici di una semantica dizionariale è quali delle marche implicate vengano negate della negazione della espressione implicitante. Cosa significa dire "colui non è scapolo"? Soltanto che non è sposato o anche che non è maschio, umano e adulto? Il buon senso ci dice che la risposta è data solo dal contesto. Se indicando un uomo dico "quell'uomo è scapolo" e il mio interlocutore mi risponde "no, non è scapolo," egli certamente intende dire che è sposato, senza con ciò negare che sia maschio, umano e adulto. In tal caso sia io che il mio interlocutore ci rifacciamo a un albero di Porfirio molto ridotto che, nell'universo degli uomini maschi adulti, distingue i non sposati dagli sposati. Se invece indicando un punk che danza in una discoteca dico "quello è scapolo" e il mio interlocutore dice "no, non è scapolo," può darsi che voglia intendere che colui non è un maschio, bensí una femmina fantasiosamente travestita. Ma in tal caso il contesto avrebbe in qualche modo specificato che siamo interessati a distinguere, nell'universo degli esseri umani in genere, i maschi dalle femmine. D'altra parte se, di un'ombra nella notte, dico che non è un cane, voglio dire che può essere un gatto, che non è un mammi- 
fero ma un uccellaccio, o addirittura che non è un animale ma una pietra? Una semantica in forma di dizionario non può concepire un dizionario assoluto e immodificabile, ma adatta le sue rappresentazioni dizionariali, e le gerarchizza, a seconda dei contesti.

Una volta però che si è accettato l'idea che le rappresentazioni semantiche dipendono dai contesti, l'idea di dizionario (che esibiva una certa purezza meccanica e sembrava adatto a costruire automi dotati di competezza semantica) crolla, e diventa piú utile l'idea di enciclopedia. Salvo che una enciclopelia non puo essere gerarchizzata ad albero. Si considerino le due frasi "non permettero mai che mia figlia vada sola di sera a casa di uno scapolo" e "figlia mia, coltivati il signor Giuseppe perché è scapolo." Nel primo caso il parlante associa al termine / scapolo / una proprietà negativa, nel secondo una proprietà positiva. Quando è che uno scapolo è raccomandabile e quando non lo è? Una enciclopedia deve tener conto di tutte le definizioni, anche quelle contraddittorie, ovvero di tutto cio che viene detto ed è stato detto di uno scapolo, specificando poi quali proprietà valgano in quali contesti. Si veda un termine come / balena /. Per noi oggi ha la proprietà di essere un mammifero, ma prima della zoologia moderna era considerata un pesce. Un dizionario che analizzi la balena come mammifero non mi permette di capire una quantità di testi antichi in cui la balena è vista come pesce tra i pesci. Una enciclopedia deve contenere una definizione di / balena / che consideri due selezioni contestuali: in contesti di tipo $\mathrm{A}$ è un pesce, mentre in contesti di tipo B è un mammifero. Il che sembra anche corrispondere abbastanza intuitivamente alla nozione editoriale corrente di enciclopedia, come strumento che mi fornisce le diverse eccezioni di un termine non solo secondo le leggi del lessico ma anche tenendo di vista le differenze storiche e culturali: una buona enciclopedia non mi dice solo che i cani sono mammiferi, ma che in certe religioni antiche erano venerati e in certi paesi costituiscono un cibo assai ghiotto. Solo sulla base di queste notizie enciclopediche posso capire una raffigurazione egizia del dio Annubio, o un racconto che si svolga in un ristorante di un porto asiatico dove si chiedono al cuoco dei cani.

È possibile fornire una rappresentazione strutturale di una enciclopedia semiotica di questo tipo, e vi provvedono in modi diversi la topologia e la teoria dei grafi. Ma si puo accettare come buona la metafora proposta da Deleuze e Gualtieri: in Rhizome, metafora vegetale, in cui alla radice arborescente si contrappone un viluppo, una rete di bulbi e tubercoli come "quando i ratti scivolano gli uni sugli altri."

Le caratteristiche di una struttura rizomatica sono le seguenti: 
(a) ogni punto del rizoma puó essere connesso (e deve esserlo) con qualsiasi altro punto;

(b) non ci sono punti o posizioni in un rizoma, non vi sono che delle linee;

(c) un rizoma può essere spezzato in un punto qualsiasi, e riprende seguendo una delle proprie linee;

(d) il rizoma è antigenealogico;

(e) il rizoma ha sempre un proprio esterno con cui fa ugualmente rizoma;

(f) un rizoma non è un calco ma una carta (l'orchidea non riproduce il calco della vespa, essa fa carta con la vespa in seno a un rizoma); la carta è aperta, può essere connessa con qualcos'altro in ogni sua dimensione, è smontabile, rovesciabile, suscettibile di continue modificazioni;

(g) una rete di alberi che si aprano in ogni direzione può fare rizoma (il che, ci pare, equivale a dire che in ogni rizoma può essere artificialmente ritagliata una rete di alberi parziali);

(h) il rizoma è acentrico e pertanto in esso le iniziative locali possono essere coordinate indipendentemente di una istanza centrale o originaria.

Forse il rizoma ha altre proprietà: ma questi accenni metaforici ci permettono di azzardare alcuni requisiti di una rappresentazione semantica in forma di enciclopedia:

a. Essa è strutturata secondo una rete di interpretanti, e cioè di definizioni che sostituiscono altre definizioni, di situazioni che chiariscono il senso di un termine (cosí come per spiegare a qualcuno il significato del segnale militare di / at-tenti! / posso mostrargli una raffigurazione di un manipolo di soldati che scattano a talloni uniti e piedi divaricati, testa alta e mani lungo la cucitura dei pantaloni), di rappresentazioni visive che spiegano espressioni verbali e viceversa (come se sotto a una raffigurazione del dio Anubi spiegassi che si tratta di una divinità egiziana), di sinonimi organizzati a catena, cosi che tra il primo e l'ultimo termine delle catene ogni possibile sinonimia sia scomparsa (si pensi alla catena "cosacco - militare zarista - armato al servizio di un imperatore samurai"), eccetera. La catena degli interpretanti fa sí che tra le proprietà (variamente descritte) assegnate a una espressione linguistica (o di altro sistema semiotico) alcune appaiono mutuamente contradditorie, e solo il contesto stabilisca quale debba essere attivata o, come si dice, topicalizzata, accordata al tema dominante del discorso. 
b. Essa assume pertanto non la forma di un albero gerarchizzato, ma di una rete o di un labirinto. Permette vari percorsi, scelte multiple, definibili sulla base dei contesti o di precise regole testuali.

c. Essa è potenzialmente infinita, perché deve tener conto di tutte le proprietà che tutte le culture hanno attribuito alle varie espressioni. Essa è potenzialmente infinita perché è mobile, e i discorsi che facciamo sulla base dell'enciclopedia di fat to mettono continuamente in questione l'enciclopedia, nel senso che l'ultimo articolo scientifico di uno scienziato nucleare prevede una serie di conoscenze enciclopediche circa la struttura dell'atomo, ma ne introduce di nuove e pone in crisi alcune di quelle vecchie.

d. Essa non è preoccupata di registrare quello che "è vero" (qualunque senso si assegni a questa espressione) ma registra invece ciò che socialmente è stato detto, e non solo quello che è stato accettato per vero, ma anche quello che è stato accettato come immaginario. Una enciclopedia non registra solo che Napoleone è morto a Sant'Elena, ma anche che Don Rodrigo è morto di peste, almeno nel mondo immaginario di Manzoni, perché anche le notizie concernenti il mondo di Manzoni fanno parte dell'enciclopedia.

L'enciclopedia semantica quindi non è mai finita, esiste come idea regolativa: e tuttavia questa idea regolativa, che non puo dare origine a un proget to editoriale, perché non è finita e perché non ha forma organizzabile, serve a individuare le porzioni identificabili dell'enciclopedia attuale, come riserva di sapere sociale e socializzato, nella misura in cui servono a costruire gerarchie provvisorie, o reti maneggiabili, ai fini di interpretare e di spiegare la interpretabilità di certe porzioni di discorso. Tutta la semantica degli ultimi anni tenta di lavorare intorno a questa nozione fantasma di enciclopedia, che pare l'unica adatta a spiegare l'uso naturale dei linguaggi e a spiegare perché e come noi comprendiamo le espressioni che riceviamo nel corso delle interazioni comunicative.

Questa nozione di enciclopedia non nega l'esistenza di un sapere: nega la sua organizzabilità in modo definito e permanente. Non nega che questo sapere possa essere indagato e organizzato "localmente"; nega che se ne possa dare organizzazione globale: ovvero asserisce che ogni organizzazione che si vuole globale, ignorando di essere parziale, è perciò stesso ideologica.

Questa enciclopedia vede le enciclopedie editoriali come sue attualizzazioni provvisorie, incomplete, parziali, buone sole per certi fini: e fatalmente ideologiche quando pretendano di essere esuastive, o identifichino l'ordine della loro organizzazione con un 
ordine "oggettivo," celando i propri criteri organizzativi, e i fini che han determinato questi criteri.

Questa enciclopedia non esiste, eppure è la totalità di ciò che l'umanità ha detto, ed ha una esistenza materiale, perché questo det to è stato depositato sulla forma di altri libri, di quadri, di film, di comportamenti, di costruzioni architettoniche, di leggi, di strade. ...

Trasformatasi da utopia della conoscenza globale (raggiungibile) in coscienza dell'impossibilità della conoscenza globale ma in sicurezza della reperibilità locale degli elementi di questa conoscenza, diventata da progetto di un libro a metodo di indagine attraverso la biblioteca generale e onnivora della cultura tutta, l'enciclopedia di quarta forma è importante proprio perché non esiste come oggetto riconoscibile. $\grave{E}$ ancora, come per Dante, ciò che per l'universo si squaderna: salvo che si tratta dell'universo della cultura e non è visibile legato in un volume. Almeno, non in uno solo.

Università di Bologna 